\title{
Electrically Short Dipoles With a Nonlinear Load, a Revisited Analysis
}

\author{
John M. Ladbury, Member, IEEE, and Dennis G. Camell, Senior Member, IEEE
}

\begin{abstract}
We reexamine the characteristics of electrically short dipoles with nonlinear loads and, specifically, the early work of Motohisa Kanda. Although this topic has been examined in great detail in the past, some inconsistencies between numerical and analytical results are apparent, and these have not been previously addressed. We show that these inconsistencies were due to only periodic sampling of the analytic solution, and an insufficient number of iterations in the numerical solutions, and we give corrected results. Additionally, some of the more significant analytical results, which were once thought to be impractical due to their complexity, are numerically implemented. We also show that a simple approximation accurately describes the behavior of these electrically short dipoles over a wide range of frequency and amplitude.
\end{abstract}

Index Terms-Analytical, diode, dipole, electric field, electrically short, nonlinear, numerical, probe.

\section{INTRODUCTION}

$\mathbf{T}$ HIS PAPER addresses some inconsistencies in the early work of Kanda [1]-[6] dealing with the analysis of an electrically short dipole with a nonlinear (diode) load. Such dipoles with diode loads are commonly used in electric-field probes. We show that Kanda's analysis (both analytical and numerical) was approximately correct over the typical operating range of a dipole probe (frequencies greater than $1 \mathrm{MHz}$ and output voltages greater than $0.1 \mu \mathrm{V}$ ). However, recent applications have forced us to examine the characteristics at lower ranges of frequency and output voltage, where the approximations and simplifications used by Kanda are no longer valid. In extreme cases, results based on invalid simplifications can show errors of greater than $20 \mathrm{~dB}$. The inconsistencies described here were still present in a recent (1995) publication [6]. We examine the sources of these inconsistencies and give corrections.

We began examining Kanda's work because we wanted to simplify the calibration of electrically short dipole probes, and Kanda had written a number of papers on this subject. In the earliest of these papers [1], Kanda analyzed an electrically short dipole with a nonlinear load using both analytical and numerical techniques. Initially, his results appeared to match the observed characteristics of our dipole probes, but two characteristics of his results were puzzling: substantial differences existed between some of the numerical and analytical solutions (especially at low frequencies and low input voltages), and the analytical solution suggested a positive bias across the diode load for low input voltages (a result that runs counter to both the nu-

Manuscript received May 1, 2001; revised August 1, 2001.

The authors are with the Radio-Frequency Technology Division, National Institute of Standards and Technology, Boulder, Colorado 80305 USA (e-mail: ladbury@boulder.nist.gov).

Publisher Item Identifier S 0018-9375(02)01457-6. merical models and engineering experience). Upon further investigation we found that, although the theoretical analysis and numerical models were sound, there were subtle errors in the implementation of both.

This paper is structured as follows. In Section II, we examine some of the results published by Kanda and point out some of the apparent problems. In Section III we discuss the sources of the differences and give corrected results. We present some of the approximations that have been used to describe the behavior of these probes in Section IV. One of these approximations is quite simple, and describes the high-frequency behavior of these probes over a wide range of input voltages. Finally, in Section V, we reiterate our conclusions that the basic analysis (both numerical and analytical) performed by Kanda was sound, but that the implementation of the final solutions introduced minor errors. We also conclude that one of the approximations given by Kanda as being valid for high frequencies and high voltages is, in fact, much more general than he indicated; the approximation appears to be valid over a wide range of frequencies (greater than $100 \mathrm{kHz}$ ) and over the entire range of input and output voltages.

\section{EARLY WORK}

Kanda used a simple Thevenin's equivalent circuit of an electrically short dipole with a diode [1], which is reproduced in Fig. 1. The driving voltage $v_{i}(t)$ is the induced open-circuit voltage at the dipole terminal and is given by

$$
v_{i}(t)=e_{\mathrm{inc}}(t) h_{e}
$$

where $e_{\mathrm{inc}}$ is the normal incident electric field strength and $h_{e}$ is the effective length of the dipole. In Fig. 1, $C_{a}$ is the equivalent driving point capacitance of the dipole, and a parallel combination of a diode capacitance $C_{d}$ and a nonlinear (diode) resistance $R_{d}$ represents a simplified model of the diode. The physical dimensions were listed as a dipole length of $2 h=0.04 \mathrm{~m}$ and an antenna radius of $a=2.84 \cdot 10^{-5} \mathrm{~m}$. Unfortunately, an error in the equations for the effective length and dipole capacitance (corrected versions are given in [6]), resulted in incorrect values for the dipole parameters used by Kanda. The dipole parameters for Kanda's model were given as $h_{e}=9.72 \cdot 10^{-3}$ $\mathrm{m}$ and $C_{a}=0.2 \cdot 10^{-12} \mathrm{~F}$. For consistency, we will use these values in our analysis. The diode's capacitance is given as $C_{d}=$ $0.34 \cdot 10^{-12} \mathrm{~F}$, and the nonlinear resistance $R_{d}$ of the diode is characterized by its $i-v$ characteristic

$$
i(t)=I_{S}\left(e^{\alpha v_{o}(t)}-1\right) .
$$




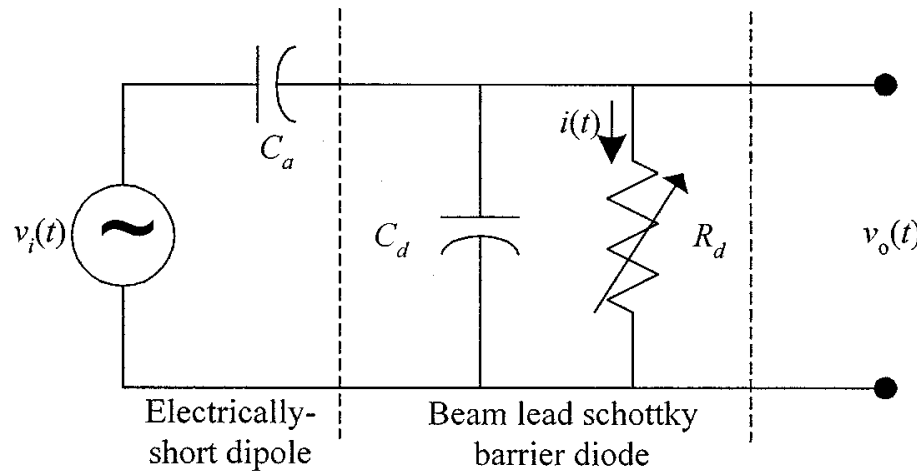

Fig. 1. Thevenin's equivalent circuit of an electrically short dipole with a diode.

In (2), $i(t)$ is the current through the nonlinear resistance, $v_{o}(t)$ is the voltage across the diode, $I_{S}=2 \cdot 10^{-9} \mathrm{~A}$ is the saturation current, and $\alpha=q / n k T \approx 38 \mathrm{~V}^{-1}$, where $q=106 \cdot 10^{-19} \mathrm{C}$ is the electronic charge, $n \approx 1.05$ is the diode ideality factor, $k=1.38 \cdot 10^{-23} \mathrm{~J} / K$ is Boltzmann's constant, and $T \approx 290 \mathrm{~K}$ is the temperature. In the analysis that follows, we will make the same assumption as Kanda: that the diode $i$ - $v$ characteristics given in (2) describe the diode behavior for all values of current and voltage. This is considered a good general model and should be adequate for our analysis, but will not describe all known diode characteristics [7].

Kanda analyzed the circuit shown in Fig. 1 both analytically and numerically for a sinusoidal input voltage $v_{i}(t)=V_{i} \sin (\omega t)$. The steady-state output voltage $v_{o}(t)$ is a periodic signal of the same period $p=2 \pi / \omega$ as the input signal. Once this signal has been filtered to remove the high frequency content, we measure the remaining dc voltage $\bar{V}_{o}=(1 / p) \int_{0}^{p} v_{o}(t) d t$, averaged over a complete cycle. The details of Kanda's analysis are given in [1]. Two plots presented in several of Kanda's papers [1]-[6] were digitized and are reproduced in Figs. 2 and 3. Fig. 2 gives the detected dc voltage $\left|\bar{V}_{o}\right|$ across the diode as a function of the induced voltage $V_{i}$ at a driving frequency of $100 \mathrm{MHz}$ for both the analytical and numerical techniques. For large induced voltages $\left(V_{i}>1 \mathrm{~V}\right)$, the numerical solution is about $45 \%$ greater than the analytical solution, and for small induced voltages the analytical solution is about seven times greater than the numerical solution. The sizes of these offsets was surprising, especially given the simplicity of the circuit model, the fact that both solutions are based on the same diode model, and the general ability of numerical models to simulate similar nonlinear circuits [7]. Fig. 3 gives the transfer function $\left|\bar{V}_{o}\right| / V_{i}$ of the circuit shown in Fig. 1. Using $e_{\mathrm{inc}}=1 \mathrm{~V} / \mathrm{m} \mathrm{rms}$ as asserted in [1] and (1) we calculate a driving voltage of $9.72 \cdot 10^{-3} \cdot \sqrt{2}=0.0137$ $\mathrm{V}$. The transfer function in Fig. 3 is approximately $-10 \mathrm{~dB}$ at $100 \mathrm{MHz}$, but this does not agree with Fig. 2, which shows that $\left|\bar{V}_{o}\right|$ is approximately $2 \cdot 10^{-4} \mathrm{~V}$, or $35 \mathrm{~dB}$ below $V_{i}$ for $V_{i}=0.0137 \mathrm{~V}$. Most likely, the actual drive voltage used to generate Fig. 3 was $V_{i}=1 \mathrm{~V}$. Further investigation showed that the apparent null near $8 \mathrm{kHz}$ in Kanda's transfer function depends on the input signal, and an input signal of $V_{i}=1 \mathrm{~V}$ results in a curve very similar to that given in Fig. 3.

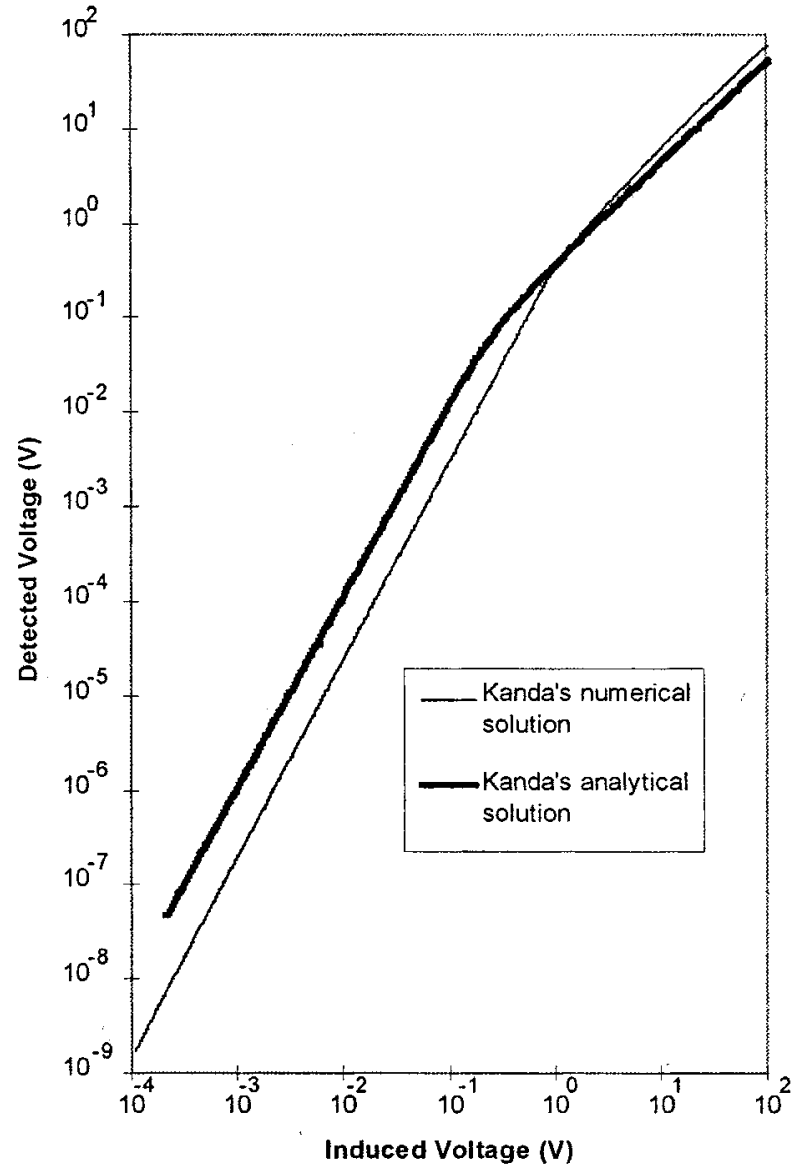

Fig. 2. Detector's voltage response at $100 \mathrm{MHz}$.

The differences between the analytical and numerical solutions of the transfer function are substantial, with differences of approximately $9 \mathrm{~dB}$ at $10 \mathrm{GHz}$, and below $7 \mathrm{kHz}$ no analytical solution was given by Kanda. Even though we do not expect typical probes to be used much below $100 \mathrm{kHz}$, the existence of a difference between these curves across the frequency spectrum emphasizes that a problem is present with one or both of the solutions. We expected that the analytical solution was correct at high frequencies, since we expected a constant transfer function. However, the falloff of the analytical solution near $7 \mathrm{kHz}$ is unexpected, and we expected the numerical solution to describe the behavior of the circuit more accurately here.

As a final puzzle, Kanda's analytical solution predicts a negative value for $\bar{V}_{o}$ (biasing the diode "off") for most large values of frequency and input voltage, but also predicts a small positive value for small values of frequency and input voltage. To show this, we use the series representation of Kanda's analytical solution [1]

$$
\bar{V}_{o}=-\frac{1}{\alpha} \ln \left[S_{1}(T, U)-T S_{2}(T, U)\right]
$$

where $T$ is the normalized period given by

$$
T=\frac{\alpha I_{s}}{\omega\left(C_{a}+C_{d}\right)}
$$




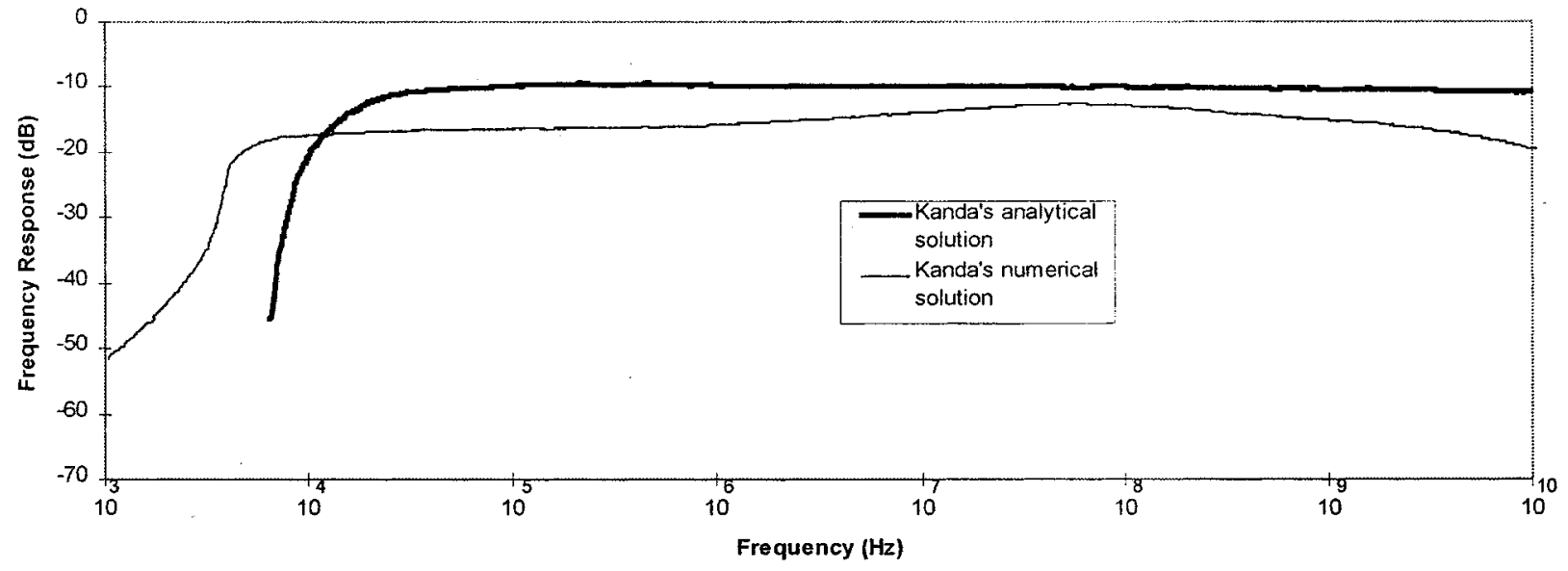

Fig. 3. Transfer function of an electrically short dipole with a nonlinear load.

$U$ is the normalized induced voltage given by

$$
U=\frac{\alpha V_{i}}{1+C_{d} / C_{a}},
$$

and the series representations $S_{1}(T, U)$ and $S_{2}(T, U)$ are

$$
S_{1}(T, U)=1+\sum_{\substack{m=2 \\ \text { cven }}}^{\infty} \frac{U^{m}}{\prod_{\substack{k=2 \\ \text { cven }}}^{m}\left(k^{2}+T^{2}\right)}
$$

and

$$
S_{2}(T, U)=\sum_{\substack{m=1 \\ \text { odd }}}^{\infty} \frac{U^{m}}{\prod_{\substack{k=1 \\ \text { odd }}}^{m}\left(k^{2}+T^{2}\right)} .
$$

Taking the first three terms of $S_{1}(T, U)-T S_{2}(T, U)$ (which dominate for small values of $U$ ), we have

$$
S_{1}(T, U)-T S_{2}(T, U) \approx 1-T \frac{U}{1+T^{2}}+\frac{U^{2}}{4+T^{2}},
$$

which has a value less than one for small values of $U$ and positive values of $T$. If the argument of (3) is less than one (but greater than zero), then $\bar{V}_{o}$ will be positive.

\section{CORRECTIONS}

We first duplicated Kanda's numerical model of the circuit in Fig. 1 to see if we could duplicate his results. Using our model, we then duplicated Kanda's simulation conditions by simulating the system for 400 periods of the input signal, with 16 sample points per cycle. Our numerical results were similar to those of Kanda, as shown in Fig. 4, with a few notable exceptions. For large input signals $\left(V_{i}>1 \mathrm{~V}\right)$, our results agreed better with the analytical solution. For small input signals $\left(V_{i}<1 \mathrm{~V}\right)$, our numerical results fell somewhere between Kanda's numerical and analytical results. Further investigation showed that our numerical model had not reached a steady-state solution after 400 periods. If we allowed the solution to become stable (which in some cases took over 4000 periods), we obtained much better agreement, as shown in Fig. 4. Further improvement was obtained by increasing the number of samples per period.

The transfer function generated by our numerical model showed poorer agreement with Kanda's, as shown in Fig. 5.

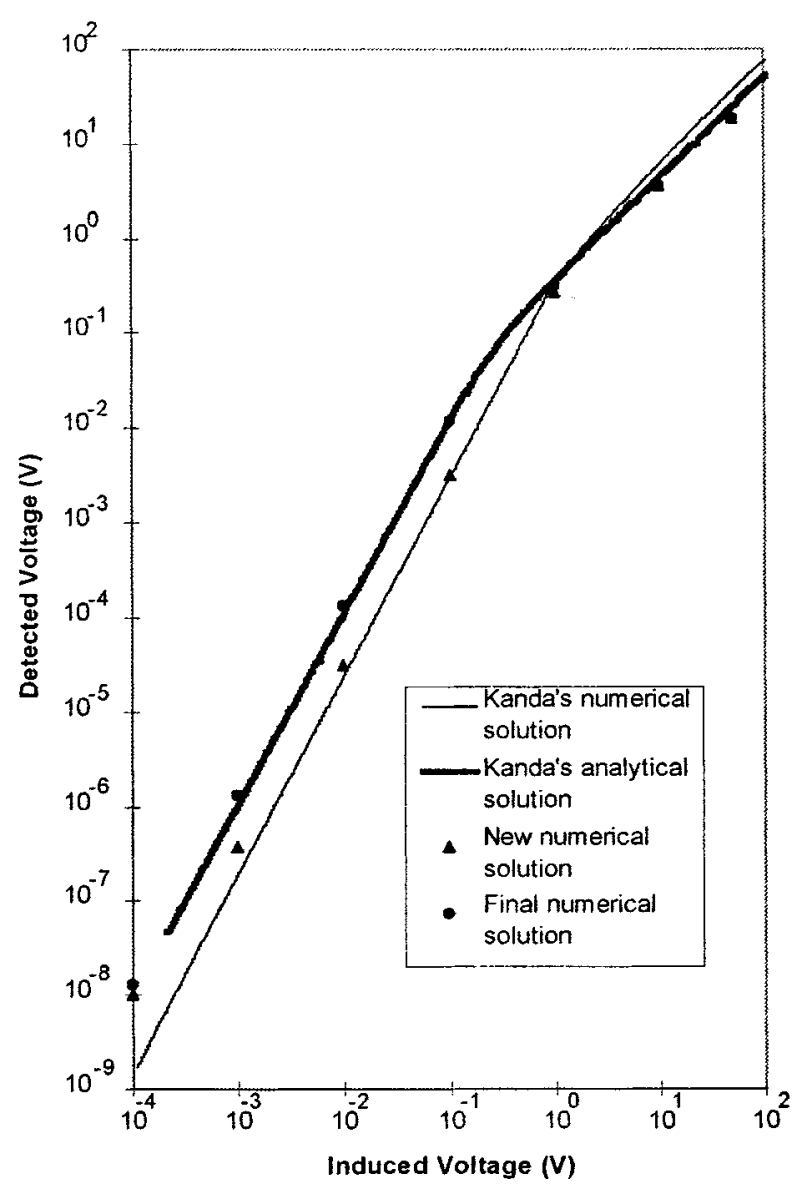

Fig. 4. Detector's voltage response at $100 \mathrm{MHz}$. Also shows attempts to duplicate Kanda's numerical solution.

We showed similar behavior above $100 \mathrm{MHz}$, but our low-frequency behavior showed substantial differences, and we were unable to duplicate Kanda's results. Allowing our model to reach steady-state conditions helped improve the agreement between the analytical and numerical solutions for frequencies above $20 \mathrm{kHz}$, but still showed a large difference at lower frequencies. We assumed that the difference must be due to an error in the analytical solution. 


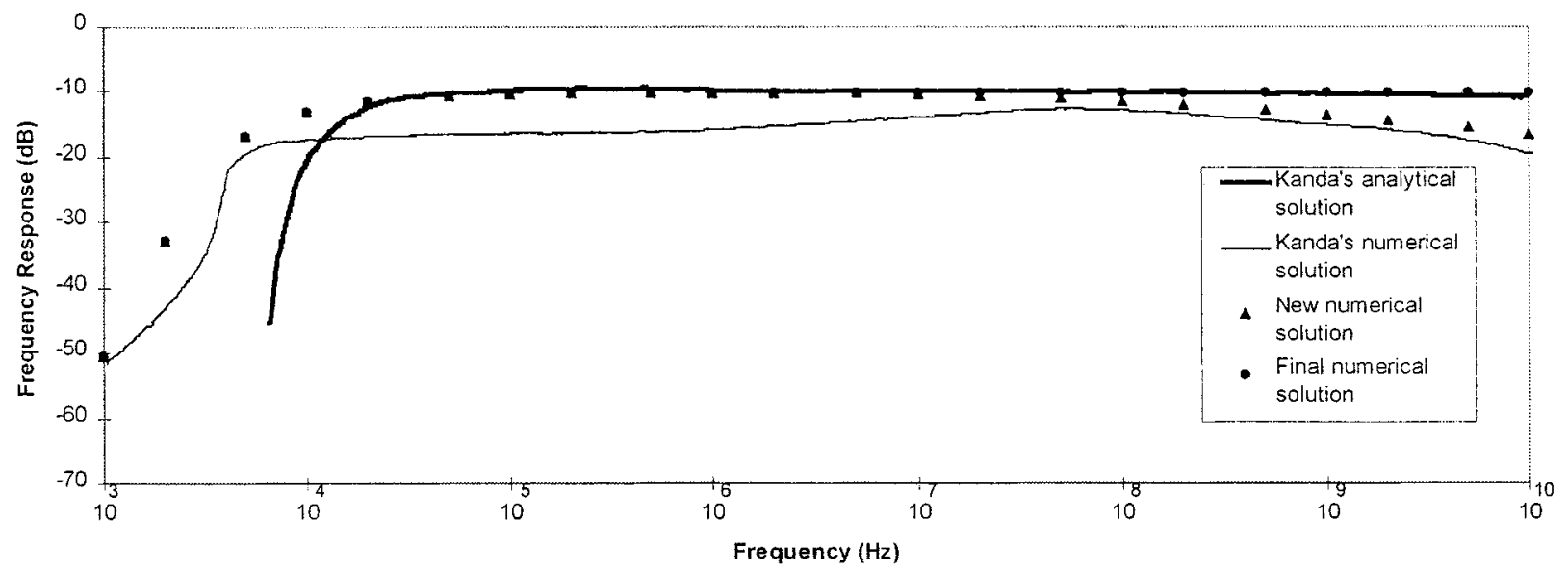

Fig. 5. Transfer function of an electrically short dipole with a nonlinear load. Also shows attempts to duplicate Kanda's numerical solution.

Kanda derived the following analytical equation for the output voltage

$$
v_{o}(t)=\frac{b v_{i}(t)}{\alpha}-\frac{1}{\alpha} \ln \left[d e^{-a t}+a e^{-a t} \int_{0}^{t} e^{b v_{i}(\tau)+a \tau} d \tau\right]
$$

where

$$
\begin{aligned}
& a=\frac{\alpha I_{S}}{C_{a}+C_{d}}=140.7 \mathrm{kHz} \\
& b=\frac{\alpha}{1+C_{d} / C_{a}}=14.1 \mathrm{~V}^{-1}
\end{aligned}
$$

and $d$ is undefined in Kanda's paper. We assumed $d$ was equal to 1 so that (9) goes to 0 at $t=0$ (this assumption is unimportant since this term vanishes for large $t$ ). We compared the numerical and analytical solutions as a function of time and observed very similar results, so there did not appear to be an error in (9). However, Kanda then shows how to compute (9) for time $t=n p$ equal to integer multiples of the period. This is simply a sample of the output, not the average of the output over one cycle. This becomes a good estimate of the average over one cycle if the dc component of the output is large, which is probably why Kanda did not notice the problem. But, for small values of input voltage, the output voltage is sometimes positive and sometimes negative, and, therefore, periodic samples can also be either positive or negative, even if the dc component is strictly negative. A more general approach is given by Randa and Kanda [8], but it still gives the instantaneous output voltage as a function of time, rather than the average over one period. Their result, which is valid for steady-state conditions, was

$$
\begin{aligned}
& v_{o}(t)=\frac{b v_{i}(t)}{\alpha}-\frac{1}{\alpha} \ln \left\{a e^{-x p a}\left[\frac{1}{e^{a p}-1} I(1)+I(x)\right]\right\} \\
& I(x) \equiv \int_{0}^{x p} e^{b v_{i}(\tau)+a \tau} d \tau
\end{aligned}
$$

where $0 \leq x<1$. Averaging (12) over one period gives us

$$
\bar{V}_{o}=-\frac{1}{\alpha} \int_{0}^{1} \ln \left\{a e^{-x p a}\left[\frac{1}{e^{a p}-1} I(1)+I(x)\right]\right\} d x
$$

where the first term in (12) vanishes because we assume the input signal does not have a dc component. We could not find a simple closed-form solution for (13), but it is well behaved and simple to compute numerically. Fig. 6 shows the transfer function described by (13) for various values of the input voltage. The variability in the high-frequency results for $V_{i}=1 \mu \mathrm{V}$ is due to limited resolution of the numerical computation of (13). In all cases, the transfer functions are essentially flat for frequencies greater than $100 \mathrm{kHz}$. This is confirmed in Fig. 7, which shows the detector's voltage response at various frequencies. In all cases, the numerical and analytical solutions agree and are, therefore, indistinguishable in the figure.

\section{APPROXIMATIONS}

Since both Kanda's original solution and (13) are difficult to solve, several attempts have been made to approximate the detector's voltage response as a function of input voltage. Kanda showed that, for small input voltages, the output voltage was proportional to the square of the input voltage

$$
\bar{V}_{o} \approx-\frac{\alpha}{4}\left[\frac{V_{i}}{1+C_{d} / C_{a}}\right]^{2}
$$

and for large input voltages, the output voltage is directly proportional to the input voltage:

$$
\bar{V}_{o} \approx-\frac{V_{i}}{1+C_{d} / C_{a}} .
$$

Equations (14) and (15) can be combined to give a continuous approximation of $\bar{V}_{o}$ as a function of $V_{i}$ of the form $c_{1} V_{i}^{2} /\left(c_{2}+\right.$ $V_{i}$ ) where the constants $c_{1}$ and $c_{2}$ are chosen such that the behavior agrees with (14) for small input voltages, and with (15) for large input voltages. The combined solution is

$$
\bar{V}_{o} \approx-\frac{1}{1+C_{d} / C_{a}} \cdot \frac{V_{i}^{2}}{4\left(1+C_{d} / C_{a}\right) / \alpha+V_{i}}
$$

The high-frequency analytical solution, along with the square-law approximation in (14), the linear approximation in (15), and the combined approximation in (16) are all plotted in Fig. 8. These approximations have several desirable characteristics, two of which are the possibility of extrapolation 


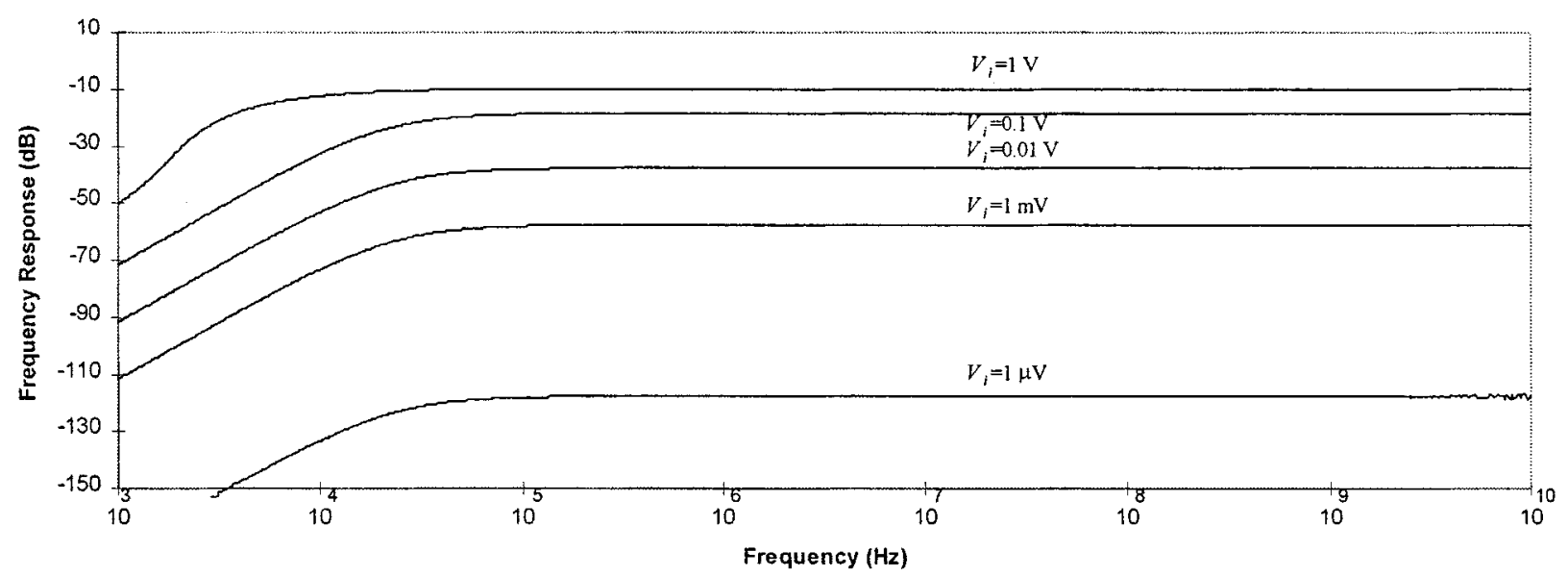

Fig. 6. Corrected transfer function of an electrically short dipole with a nonlinear load for various input voltages. Both analytical and numerical results agree.

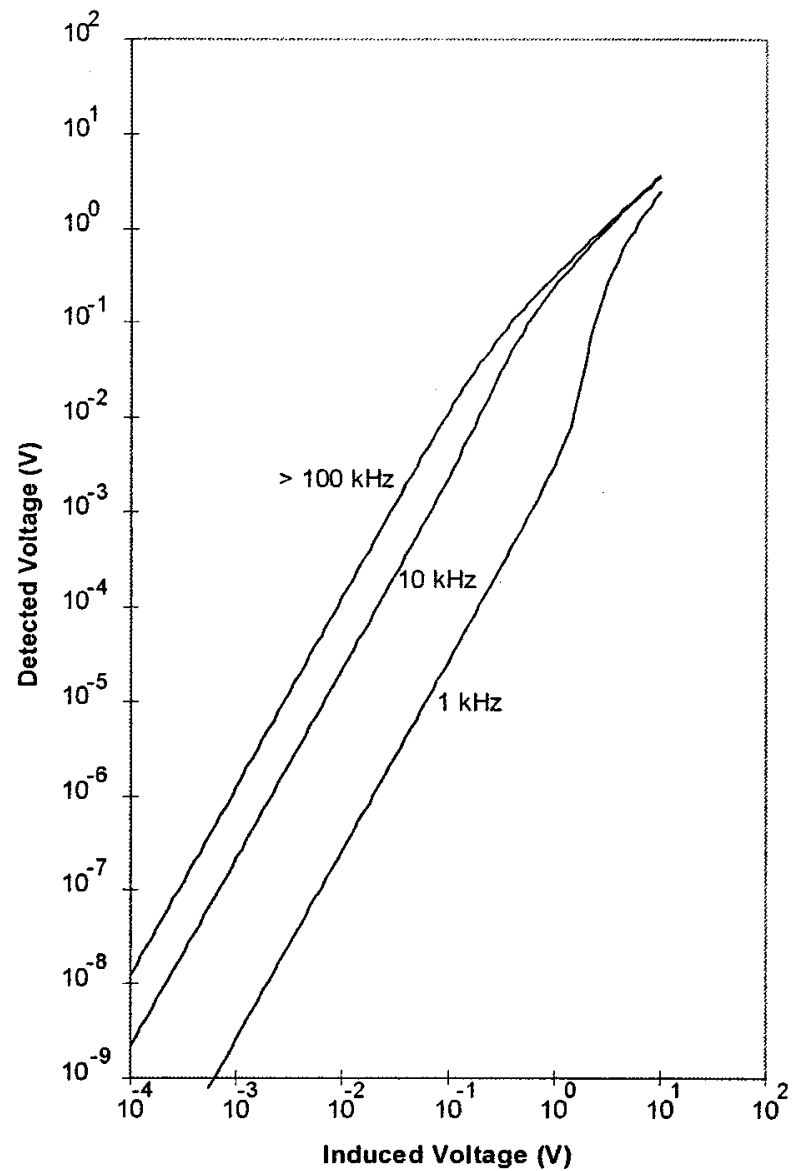

Fig. 7. Corrected detector's voltage response at various frequencies. Both analytical and numerical results agree and are indistinguishable in this figure.

and inversion. Assuming that the circuit model is correct but that slight variations in component parameters $\left(C_{d}, C_{a}, \alpha\right)$ are possible, then a calibration over a small range of $V_{i}$ and $\bar{V}_{o}$ can easily and accurately be extrapolated to give useful results outside of the calibration range. (However, extrapolation is always a risky proposition and should be used with great caution, since small imperfections in the circuit model can result in large errors. Some sort of limited verification is recommended before extrapolated results are accepted.) Inversion is also useful because, although calibrations are generally performed by varying the incident electric field (which is proportional to $V_{i}$ ), and then measuring $\bar{V}_{o}$, the final application of such probes involves measuring output voltage and then calculating the incident electric field. This implies that the relationship between the input and output voltages should be invertable, which is the case for (14)-(16).

Another approximation is also provided by Kanda. He states that, for large $V_{i}$, the dc voltage averaged over a complete cycle becomes

$$
\bar{V}_{o} \approx-\frac{1}{\alpha} \ln I_{0}\left(\frac{\alpha V_{i}}{1+C_{d} / C_{a}}\right)
$$

where $I_{0}(\cdot)$ is the modified Bessel Function of zeroth order. Based on Kanda's caveat that this approximation is good for large $V_{i}$, we did not expect this approximation to be much more useful than that given in (15). However, after implementing routines for computing $I_{0}(\cdot)$ [9] and its inverse (details are given in Appendix), we found that (17) appeared to be a good approximation for all values of $V_{i}$. This relationship still needs to be verified analytically, but plots of our final high-frequency analytical and numerical solutions were indistinguishable from plots of (17), so there is little reason to show the result here. The general applicability of (17) could simplify the analysis of dipole probes described by Kanda. It is unfortunate that one of the most significant results of Kanda's analysis has not had greater exposure.

\section{CONCLUSION}

We have examined Kanda's early work on electrically short dipoles with nonlinear (dipole) loads, and have shown that the basic analysis is sound and reliable. We have also shown that the approximations used by Kanda are valid over the typical operating range of common electric-field probes. However, both the numerical and analytical approaches used by Kanda give erroneous results for small values of input voltage and frequency. We showed that the error in the analytical solution was due to the fact that Kanda periodically sampled the output voltage rather than averaging over a full period. Kanda's sampling procedure was shown to be a good approximation of the ideal result 


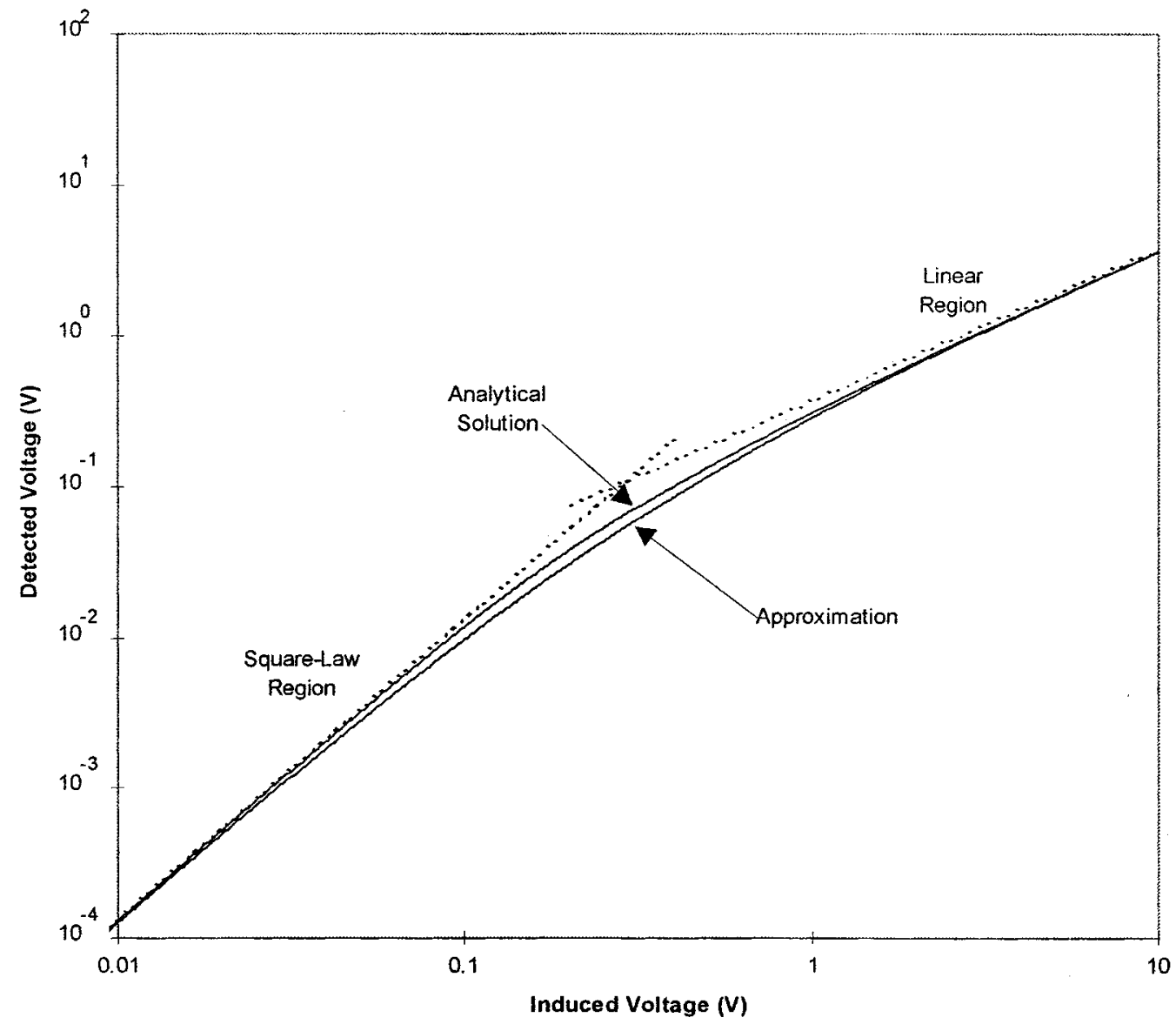

Fig. 8. High-frequency analytical solution, along with linear, square-law, and combined approximations.

for moderate to large input voltages, and this is most likely the reason that Kanda did not detect the problem.

We also showed that Kanda's numerical procedure can give accurate results, but that some of the results published by Kanda were based on a simulation that had not yet converged to a final solution. Allowing the procedure to continue until it converged to a solution and also sampling at a higher resolution allowed us to see consistent agreement between the analytical and numerical solutions.

Finally, we showed that one of the approximations given by Kanda, that the output voltage can be computed using a natural logarithm and a zeroth-order modified Bessel Function, is quite good at high frequencies and over a wide range of input voltages. Since the zeroth-order modified Bessel function can be computed and inverted using simple procedures, this approximation is both simple and powerful, and will probably prove to be much more useful than Kanda realized.

\section{APPENDIX}

\section{A. Approximations and Procedures for Computing the $\ln I_{o}(x)$} and Its Inverse

The modified Bessel Function of order zero $I_{0}(x)$ has been studied in detail by Abramowitz and Stegun [9], and they give several powerful approximations. For large arguments, $I_{0}(x)$ is proportional to $e^{x} / \sqrt{x}$. This can lead to computational problems for very large $x$ (greater than 700), so we recommend first applying the natural logarithm to each product term of the approximations given in [9], rather than computing $I_{0}(x)$ directly and then computing the logarithm.

There is no direct formula for inverting either $I_{0}(x)$ or $\ln I_{0}(x)$. However, a simple iterative procedure based on the Newton-Raphson method [10], and implemented below in (A1), will quickly converge to a solution. In general, given a function of the form $y=f(x)$, where $y$ is known and we would like to determine $x$, we begin with an initial guess $x_{0}$ and then iterate using

$$
x_{i+1}=x_{i}-\frac{f\left(x_{i}\right)-y}{f^{\prime}\left(x_{i}\right)} .
$$

As long as the initial guess $x_{0}$ is good, this procedure will generate an accurate solution very quickly. If we choose $f(x)$ to be $\ln I_{0}(x)$, then $f^{\prime}(x)=I_{1}(x) / I_{0}(x)$, and all that is left is to choose an initial guess $x_{0}$. Using an approach similar to that used to obtain (16), and based on the asymptotic expansion of $\ln I_{0}(x)$ for small and large $x$, a good approximation is

$$
y=\frac{x^{2}}{4+x} .
$$

Inverting (A2) gives us an initial guess of

$$
x_{0}=\frac{y+\sqrt{y^{2}+16 y}}{2} .
$$


Using (A3) as a starting point, we can iterate using

$$
x_{i+1}=x_{i}-\left(\ln I_{0}\left(x_{i}\right)-y\right) \frac{I_{0}\left(x_{i}\right)}{I_{1}\left(x_{i}\right)} .
$$

For large values of $x_{i}, \ln I_{0}(x)$ should be simplified as described above. Similar approximations are available for $I_{1}(x)$ [9], and $I_{1}(x)$ has an asymptotic expansion identical to that of $I_{0}(x)$ for large $x$. Therefore to avoid overflow problems, $I_{0}\left(x_{i}\right) / I_{1}\left(x_{i}\right)$ should be simplified by factoring out the asymptotic terms before computing the ratio.

\section{REFERENCES}

[1] M. Kanda, "Analytical and numerical techniques for analyzing an electrically short dipole with a nonlinear load," IEEE Trans. Antennas Propogat., vol. 28, pp. 71-78, Jan. 1980.

[2] — , "Analyzing short dipoles with nonlinear loads," Microwaves \& $R F$, vol. 22, pp. 74-77, 1983.

[3] M. Kanda, E. B. Larson, M. Borsero, P. G. Galliano, I. Yokoshima, and N. S. Nahman, "Standards for electromagnetic-field measurements," in Proc. IEEE, Jan. 1986, vol. 24, pp. 120-128.

[4] M. Kanda, "Standard probes for electromagnetic-field measurements," IEEE Trans. Antennas Propogat., vol. 41, pp. 1349-1364, Nov. 1993.

[5] — " "Standard antennas for electromagnetic-interference measurements and methods to calibrate them," IEEE Trans. Eletromagn. Compat., vol. 36, pp. 261-273, May 1994.

[6] - "Methodology for electromagnetic-interference measurements," IEICE Trans. Communicat., vol. E78B, pp. 88-108, 1995.

[7] P. W. Tuinenga, SPICE: A Guide to Circuit Simulation and Analysis Using PSpice. Englewood Cliffs, NJ: Prentice-Hall, 1988.

[8] J. Randa and M. Kanda, "Multiple-source, multiple-frequency error of an electric-field meter," IEEE Trans. Antennas Propogat., vol. 33, pp. 2-9, Jan. 1985.

[9] Handbook of Mathematical Functions, Applied Mathematics Series, vol. 55, National Bureau of Standards, Washington, DC, Dec. 1972. Tenth printing.

[10] W. Press, B. Flannery, S. Teukolsky, and W. Vetterling, Numerical Recipes in C: The Art of Scientific Computing. Cambridge, MA: Cambridge Univ. Press, 1988.

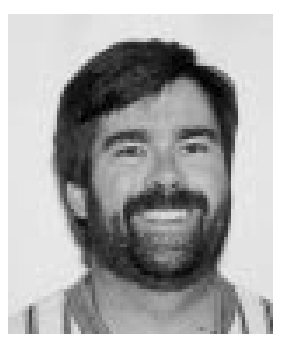

DO160D.

Mr. Ladbury is a member of the IEC joint task force on reverberation chambers. He has received two "best paper" awards at IEEE International EMC symposia.

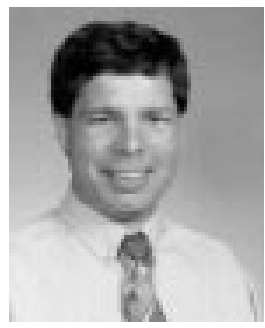

Dennis G. Camell (M'98-SM'01) received the B.S. and M.E. degrees in electrical engineering from the University of Colorado, Boulder, in 1982 and 1994, respectively.

From 1982 to 1984, he worked for the Instrumentation Directorate at White Sands Missile Range, NM. Since 1984, he has worked on probe calibrations and EMI/EMC measurements with the Radio Frequency Technology Division of N.I.S.T., Boulder, CO. His current interests are measurement analysis (including uncertainties) in various environments, such as OATS and anechoic chamber, and development of transfer standards between these environments.

Mr. Camell is involved with several EMC working standards committees. 